\title{
Distortion in Linearized Electrooptic Modulators
}

\author{
William B. Bridges, Fellow, IEEE, and James H. Schaffner, Member, IEEE
}

\begin{abstract}
Intermodulation and harmonic distortion are calculated for a simple fiber-optic link with a representative set of link parameters and a variety of electrooptic modulators: simple Mach-Zehnder, linearized dual and triple Mach-Zehnder, simple directional coupler (two operating points), and linearized directional coupler with one and two dc electrodes. The resulting dynamic ranges, gains, and noise figures are compared for these modulators. A new definition of dynamic range is proposed to accommodate the more complicated variation of intermodulation with input power exhibited by linearized modulators. The effects of noise bandwidth, preamplifier distortion, and errors in modulator operating conditions are described.
\end{abstract}

\section{INTRODUCTION}

$\mathbf{E}$ LECTROOPTIC modulators, both discrete interference types such as the Mach-Zehnder modulator and distributed interference types such as the directional-coupler modulator, have inherently nonlinear transfer curves. As a consequence, they may limit the dynamic range of the photonic link in which they are embedded by generating harmonics and intermodulation products. Various modulator configurations have been proposed and demonstrated in the last several years [1]-[8] to address this problem and increase the link dynamic range. All of these schemes depend on generating two or more modulation samples with different ratios of signal to distortion and then combining the samples so that the distortions cancel (to some order) while the signals do not cancel. In some cases it is easy to identify where the two modulations occur and where the combinations take place, as in the dual Mach-Zehnder schemes [1], [2], [6]; in others it is not so obvious, such as the directional-coupler modulator and its variations [3]-[5].

The various linearized modulator schemes predict, and in some cases have demonstrated [1], [4]-[7], significant reduction in harmonics and intermodulation products, which should lead to the realization of photonic links with higher dynamic ranges. However, in all cases, the cancellation turns out to be critically dependent upon the modulator device parameters, so that these parameters will likely have to be controlled by active means, especially if the distortion cancellation is to be maintained over a large operating bandwidth. In addition, the dependence of the harmonic or intermodulation product on the signal drive level is no longer a simple constant exponent

Manuscript received January 9, 1995; revised May 5, 1995. This work was supported in part by Contract no. F30602-91-C-0104 to Hughes Research Laboratories from the US Air Force, Rome Laboratories (N. P. Bernstein technical monitor) and by the ARPA Technology Reinvestment Project on Analog Optoelectronic Modules, Agreement No. MDA972-94-3-0016.

W. B. Bridges is with the California Institute of Technology, Pasadena, CA 91125 USA.

J. H. Schaffner is with Hughes Research Laboratories, Malibu, CA 90265 USA.

IEEE Log Number 9413707.

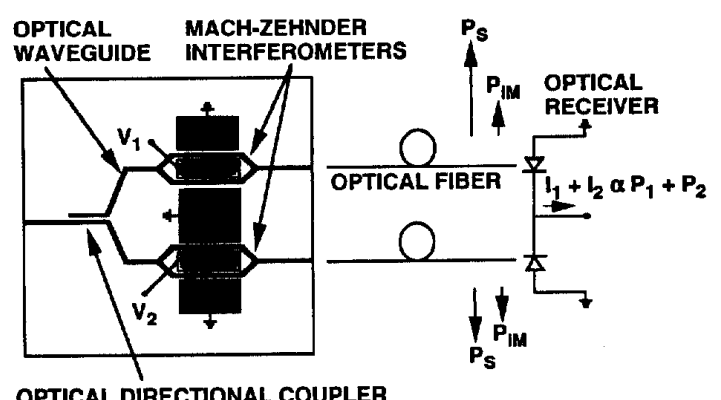

OPTICAL DIRECTIONAL COUPLER

Fig. 1. Dual-parallel modulator configured with equal length electrodes and one input optical signal. This particular approach requires two photodiodes at the optical receiver. An alternative approach would use two lasers and then combine the optical signals at the modulators' outputs into one detector

(e.g., a slope 3 line on the $\mathrm{dB}_{\text {out }}$ versus $\mathrm{dB}_{\text {in }}$ graph for thirdorder intermodulation), and the photonic link dynamic range no longer depends on the noise level in a simple way; a clearer definition of "dynamic range" is really required. Finally, the improved modulator dynamic range can easily be eroded by the nonlinear behavior of the electronic amplifiers required by the photonic link to realize reasonable gain and noise Fig. [9].

This paper uses a simple photonic link model to find the gain, noise figure, harmonics, intermodulation, and dynamic range for a number of the modulator schemes listed above, and it uses the model to optimize the modulator parameters. The sensitivity of representative Mach-Zehnder modulator (MZM) and directional coupler modulator (DCM) schemes to modulator and link parameters are calculated and compared. A refined definition of "dynamic range" is proposed to eliminate possible ambiguities resulting from the definition based on simple slopes. Finally, the results of adding electronic amplifiers to the photonic link are calculated.

\section{Il. Dual MaCh-Zehnder Modulators}

The Mach-Zehnder modulator is a simple two-channel interference device, resulting in a sine-squared dependence of light output on drive voltage. The modulator is biased to the most linear portion of the transfer curve, which for a perfect modulator also assures no even-harmonic generation.

However, the nonlinearity of the transfer curve is responsible for the generation of all odd-harmonics and all possible intermodulation products. The dual MZM scheme uses two MZM's, driven at different RF levels and fed with different optical powers, as illustrated in Fig. 1. The RF and optical power splitting ratios are chosen so that the modulator receiving the larger optical power receives the smaller RF drive power. This modulator may be thought of as the "main" modulator, with 
some distortion created by the finite RF drive power. The other modulator receives only a little optical power, but is driven relatively much harder, thus yielding a much more distorted signal. The two optical outputs are combined incoherently, for example, by combining the electrical outputs of two separate detectors as shown in Fig. 1.' If the bias points of the two modulators are chosen so that the modulations are out of phase, and the ratios of both optical and RF powers are properly chosen, then the sum of the two distortions $\left(P_{I M}\right)$ can exactly cancel, while the signals $\left(P_{S}\right)$ do not completely cancel. This exact cancellation can only occur for a specific drive level, with distortion reappearing at both lower and higher drive levels.

There are various strategies to determine the optimum ratio of optical and RF power splits to maximize the dynamic range. One strategy, first proposed and demonstrated by Johnson and Rousell [10], was arrived at by expanding the distorted output signal of each modulator in a Fourier series including the signal, odd harmonics, and intermodulation products. The coefficients in this well-known series are the products of Bessel functions. If the input signal consists of equal amplitudes at two frequencies $\omega_{1}$ and $\omega_{2}$, then the coefficient giving the intermodulation at frequency $2 \omega_{1}-\omega_{2}$ contains the product of Bessel functions $J_{1}(\theta) J_{2}(\theta)$, where the argument $\theta$ is proportional to the RF drive voltage. Johnson and Rousell then approximated this product with the first terms in the power series expansions of $J_{1}(\theta)$ and $J_{2}(\theta)$, so that the coefficient is proportional to the RF voltage cubed. To cancel this coefficient in the summed output of two modulators, they found that the optical power split ratio should be the inverse cube of the $\mathrm{RF}$ drive voltage split ratio. In their particular experiment, the RF voltage split was fixed at $1: 3$, so that the optical power split was set to $27: 1 .^{2}$ Although this particular condition cancels the cubic term in the Bessel function expansion, there remain $5^{\mathrm{th}}, 7^{\mathrm{th}}, 9^{\mathrm{th}}, \cdots$ power terms in the $\mathrm{RF}$ modulation. Thus, the intermodulation at $2 \omega_{1}-\omega_{2}$ is not exactly canceled, but exhibits a roughly $5^{t_{1}}$ power dependence on $P_{i n}$. This is illustrated in Fig. 2, which shows the intermodulation in a dual MZM with the inverse cubic relation prescribed by Johnson and Rousell. (The method of calculation and link parameters used are discussed in detail in the link model section, and in the Appendix.) The resulting dynamic range is $126.2 \mathrm{~dB}$ for this particular link, which has its component parameters given in Table I. An RF voltage split of 2.62 rather than 3 was used as discussed later.

Alternatively, the intermodulation distortion may be exactly canceled using a slightly different optical or RF splitting ratio, but only for a single power level, as illustrated by the null in Fig. 3. Slight adjustments of the splits move the exact position of the zero. The slope just to the right of the zero is steeper

\footnotetext{
Alternately, a $90^{\circ}$ polarization could be added to one output if a single detector is desired or the two modulators could be driven by two independent lasers with the receiver, comprised of a single detector.

2 Johnson and Rousell's "dual MZM" was actually a single MZM on $x$ cut $\mathrm{LiNbO}_{3}$ with the light polarized before entering the modulator such that 27 times as much optical power was in the TM polarization as in the TE polarization. A single set of electrodes modulate both optical polarizations, but the TE state is three times as sensitive to the drive voltage, as fixed by the elcctrooptic properties of lithium niobate.
}

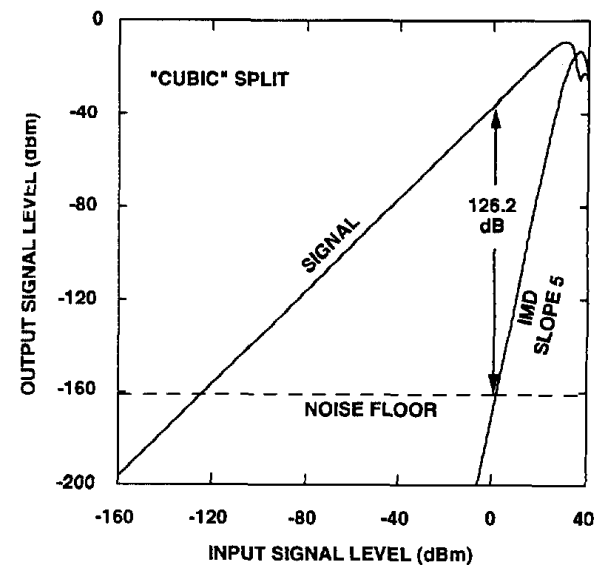

Fig. 2, Output RF signal power and third-order intermodulation power as a function of the input signal power for a fiber-optic link, with the parameters in Table I. The dual-parallel modulator is arranged for the "optimum" split so that the small-signal cubic intermodulation terms cancel, leaving a residual intermodulation at $2 w_{1}-\omega_{2}$ that varies as the fifth power of the input signal level.

TABLE I

Fiber-Oftic Link Common Parameters

\begin{tabular}{|c|c|c|c|}
\hline Laser Power & $P_{\mathbf{L}}$ & 0.1 & $\mathbf{W}$ \\
\hline Laser Noise & $\mathbf{R W N}$ & -165 & $\mathrm{~dB}$ \\
\hline Total Optical Loss & $\mathbf{L}_{0}$ & -10.0 & $\mathbf{d B}$ \\
\hline Modulator Sensitivity & $V_{S}$ or $V_{\pi}$ & 10 & $\mathbf{V}$ \\
\hline Modulator Impedance & $\mathbf{R}_{\mathbf{M}}$ & 50 & $\boldsymbol{\Omega}$ \\
\hline Detector Responsivity & $\eta \mathrm{D}$ & 0.7 & $\mathbf{A} \mathbf{W}$ \\
\hline Detector Load & $\mathbf{R}_{\mathbf{D}}$ & 50 & $\mathbf{\Omega}$ \\
\hline Noise Bandwidth & BW & 1 & $\mathrm{~Hz}$ \\
\hline Combination & $P_{2} \mathbf{L}_{0} \eta_{D}$ & 7 & $\mathbf{m A}$ \\
\hline
\end{tabular}

than 5 , while the ultimate slope to the left of the auxiliary maximum is 3 . Note that it is now possible for the IMD curve to have three intersections with the noise level line. We must specify which intersection to use to define "dynamic range." There will be no ambiguity if we define the spuriousfree dynamic range as that distance in $\mathrm{dB}$ from the signal to the intermodulation level where the intermodulation level equals the noise level at the smallest input level. With this definition, we see that the dynamic range will now depend discontinuously on the noise level. The maximum dynamic range occurs when the auxiliary maximum to the left of the minimum is just below the noise level, and the dynamic range will drop discontinuously when that maximum increases above 


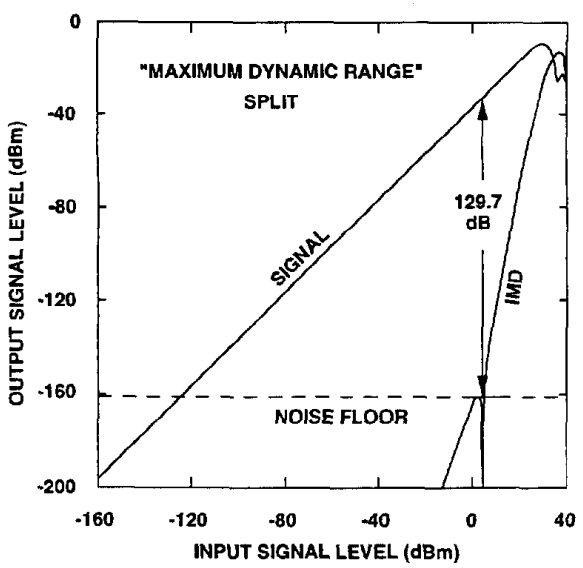

Fig. 3. Same modulator as Fig. 2 but the splitting ratio is adjusted for maximum dynamic range, which results in complete cancellation of the large-signal $2 \omega_{1}-\omega^{\prime}$ intermodulation term at one particular signal level.

the noise level. The maximum dynamic range of this link is now $129.7 \mathrm{~dB}$, compared to $126.2 \mathrm{~dB}$ for the "cubic" condition in Fig. 2. One important consequence of the more complicated behavior of the IMD and harmonics is that we must now treat the whole photonic link rather than analyze just the modulator to determine the dynamic range, since the dynamic range depends on the relationship of the noise level to the kinks and bends in the harmonic and IMD curves. The best adjustment of the modulator parameters will depend on the actual values of the other link parameters.

There is an additional degree of freedom in the true dual MZM. The condition discussed by Johnson and Rousell specifies the ratio of optical split in terms of the RF split to cancel the cubic contribution to the intermodulation. But the RF split ratio can be specified independently if a true dual MZM is used as in Fig. I instead of the two polarization states of a single modulator, where the equivalent voltage ratio is fixed at 3 . The true optimum in the voltage ratio is about 2.62 , but only one $\mathrm{dB}$ in dynamic range is sacrificed in the example given in Fig. 2 if the ratio is 1.8 or 4.8 . However, as shown later, the dynamic range is very rapidly degraded if the voltage and optical power are not near the inverse cube relation.

\section{LiNeARIZED DIRECTIONAL COUPLER MODUlators}

Integrated-optic directional couplers made on electrooptic substrates can also be used as optical modulators [11]. If the guides are physically identical, then complete transfer of the optical input from guide 1 to guide 2 is possible in one coupling length, which is determined by the optical waveguide dimensions and refractive indices of the guide and substrate. Modulating electrodes are applied to the two waveguide channels so that the propagation constants of the guides are changed incrementally in opposite directions when a voltage is applied. The differential change in the propagation constants, $\Delta \beta$, depends upon the electrode configuration and the electrooptic coefficient of the modulator material. By applying sufficient voltage, the optical signal may be transferred from guide 2 back to guide 1 . The voltage required to do this is termed

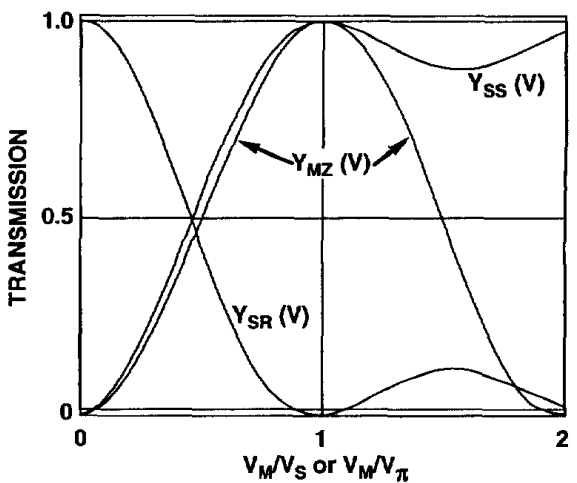

Fig. 4. Transfer curves of simple directional coupler and Mach-Zehnder modulators from zero voltage to twice the switching voltage applied to the electrodes.

the transfer voltage $\left(V_{S}\right)$, and is analogous to the half-wave voltage of the MZM. Fig. 4 shows the theoretical modulation transfer functions for a directional coupler modulator (DCM); there are two complementary transfer functions $Y_{S R}(V)$ and $Y_{S S}(V)$ since the DCM has two output channels for an input into one arm. The MZM transfer curve $Y_{M Z}(V)$ with a half wave voltage $V_{\pi}$ equal to the DCM transfer voltage $V_{S}$ is also shown for comparison. The two modulator transfer curves are very much alike from zero up to one switching voltage, but beyond that they depart; the MZM is periodic in $2 V_{\pi}$, while increasing $\Delta \beta$ further spoils the transfer from one arm back to the other. The mathematical form of the DCM transfer function [12] is

$$
Y_{S R}(V)=\frac{\sin ^{2}\left[\kappa \ell \sqrt{1+3\left(\frac{V}{V_{S}}\right)^{2}}\right]}{1+3\left(\frac{V}{V_{S}}\right)}
$$

The transfer voltage $V_{S}$ is defined by

$$
\frac{V_{S}}{3}=\frac{4 \kappa^{2} g^{2} \lambda^{2}}{\pi^{2} \zeta^{2} n_{o}^{6} r^{2}}
$$

where $l$ is the length of the coupling region and $\kappa$ is the coupling constant. When $V=0$ and $\kappa l=\pi / 2$, the signal is transferred completely from one guide to the other. The other variables in (2) are $n_{o}$ the optical index of refraction for the guide, $r$ the relevant electrooptic coefficient, $g$ the electrode gap spacing, $\zeta$ the overlap integral between the optical and electrical fields, and $\lambda$ the free space optical wavelength. $V_{S}$ is usually determined experimentally. Unfortunately, a Fourier series for the output from a modulator with this transfer function is not available in closed from. One must use a power series expansion, as in [3], or input the transfer function with a two-tone time variation and find the Fourier components numerically - as in [4] and the present work.

The intermodulation distortion produced by a simple DCM is usually very much like that of an MZM driven to produce the same modulation percentage, as pointed out by Halemane 


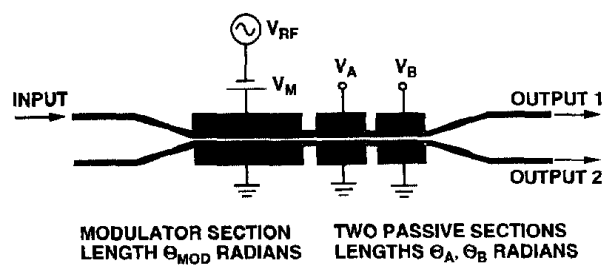

Fig. 5. Linearized directional coupler modulator with a modulator section followed by two biased passive sections. The angle $\theta$ is shorthand for $k l$.

and Korotky [12]. However, there are subtle differences. For example, biasing to the zero second-harmonic point does not eliminate higher-order even harmonics. More interesting, a zero in the third derivative curve, which is primarily responsible for both third harmonic and $2 \omega_{1}-\omega_{2} I M D$, occurs where the signal is not zero, at about $0.7954 V_{S}$. This is unlike the MZM, where zeros in all odd derivatives occur at the same value of $V_{S} / 2$. We shall return to this point later.

Attempts to linearize the transfer function given in (1) by adding elements to a basic DCM have been made by several workers [3]-[5]. Farwell et al. [4] have analyzed and built the configuration illustrated in Fig. 5, a directional coupler that has three sets of electrodes. The first set is used to apply the modulation signal plus a dc bias voltage. The second and third (passive) electrodes have only dc bias voltages applied. The two "extra" degrees of freedom introduced by these sections are used to linearize the modulation transfer function.

Before treating the modulator with three electrodes, it is instructive to look at a simpler modulator, namely a DCM with only one extra set of bias electrodes as described by Lam and Tangonan [3]. The reader may think of this as the modulator of Fig. 5 with $V_{A}=V_{B} \equiv V_{P}$ and $\theta_{P} \equiv \theta_{A}+\theta_{B}\left[\theta_{A} \equiv\right.$ $\kappa l_{A}, \theta_{B} \equiv \kappa l_{B}$ and thus $\left.\theta_{P} \equiv \kappa\left(l_{A}+l_{B}\right)\right]$. We can illustrate the development of a "more linear" region by plotting the transmission $Y_{S S}$ versus the voltage on the first section with the normalized voltage on the second section $V_{P} / V_{S}$ as a parameter. The result is shown in Fig. 6 for the particular case where both the modulator section and the biased sections are electrically $\pi / 2$ radians long: that is, $\theta_{M}=\theta_{P}=\pi / 2$. The figures give the modulation transfer curves for $-2<$ $V_{M} / V_{S}<2$, or a range of four transfer voltages. Thus, with zero voltage applied to all sections the optical input on branch 1 is completely transferred to branch 2 in $\theta_{M}$ and then back to branch 1 in $\theta_{A}+\theta_{B}$. If $V_{M} / V_{S}=1$ is applied to the modulator section with $V_{P} / V_{S}=0$, the transfer is complete from branch 1 to branch 2 . With $V_{P} / V_{S}=0$, we would bias the modulator section to $V_{M} / V_{S}=0.4394$ to obtain the minimum second harmonic output. We note that with $V_{P} / V_{S}=0.7$ applied to the second section, the region about the modulator bias point $V_{M} / V_{S} \approx 0.5$ begins to look much more linear. As the voltage is increased further, $V_{P} / V_{S}=0.8$, this added linearity disappears, and at $V_{P} / V_{S}=1$, the transfer curve is identical to $V_{P} / V_{S}=0$, but it is inverted. Further increase in the voltage applied to the second section continues to change the shape of the transfer curves but never yields such an improvement in linearity over $V_{P} / V_{S} \approx 0$. At $V_{P} / V_{S}=\sqrt{5}$, the modulation transfer curve is exactly the same as that at zero voltage, and

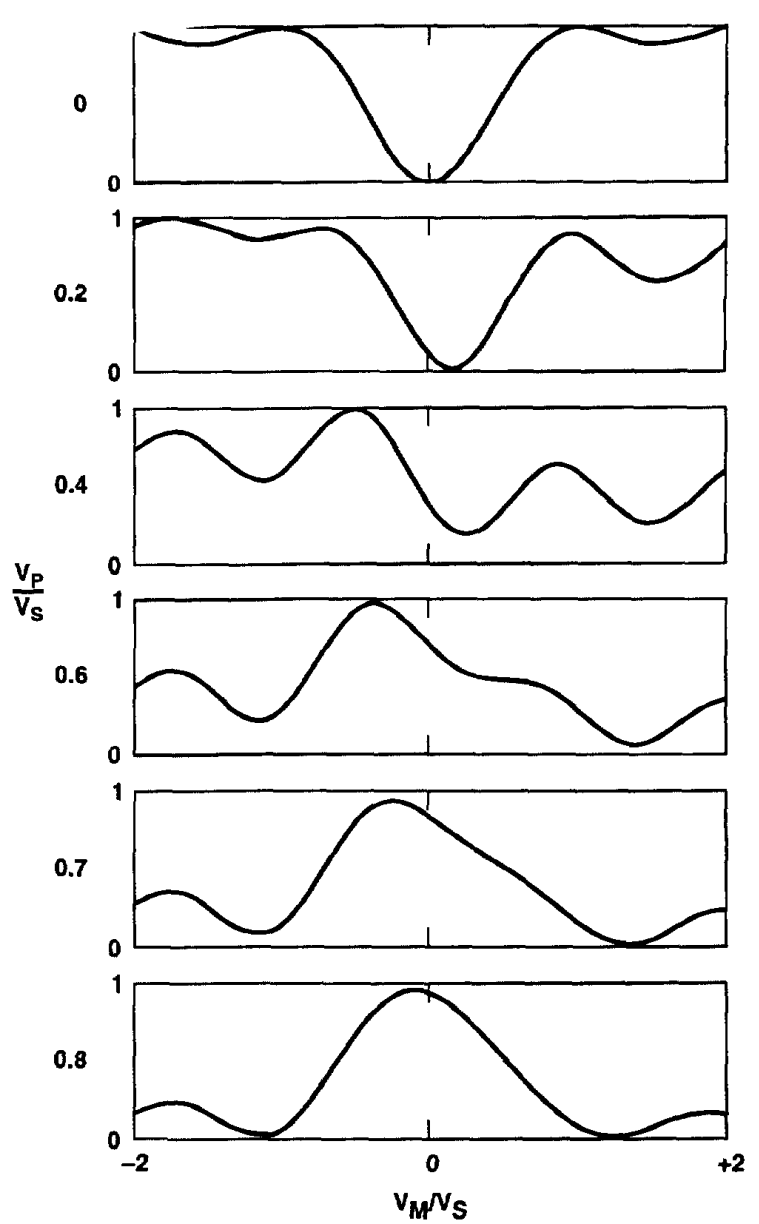

Fig. 6. Evolution of the transfer function of a directional coupler modulator with a passive bias section as the normalized voltage $V_{P} / V_{S}$ is increased from 0 to 0.8 . Note the "linearized" region on the 0.7 curve

very little change occurs above that voltage. In the limit of very high voltage applied to the second section, $\Delta \beta$ becomes so large that there is little coupling between the two guides, and the second section effectively becomes two independent guides (with equal and opposite phase shifts that still depend on the applied voltage).

It is interesting to look at the shape of the derivatives of the modulation transfer function as the bias on the second section is varied. Fig. 7 repeats the transfer function from $0<V_{M} / V_{S}<1$ and adds the first three derivatives with $V_{P} / V_{S}=0$. The first derivative produces most of the signal, the second derivative produces most of the second harmonic, and the third derivative produces most of the third harmonic and the $2 \omega_{1}-\omega_{2}$ intermodulation (and a very small amount of signal), etc. Clearly, biasing for a zero in the second derivative will nearly maximize the third derivative, an undesirable situation. What we really wish to do to is make the second and third derivatives simultaneously zero, and this can be realized if $V_{P} / V_{S}$ is changed to 0.73193 ; the resulting transfer function and its derivatives are shown in Fig. 8. This condition is near the "0.7" curve in Fig. 6. By making the second derivative 

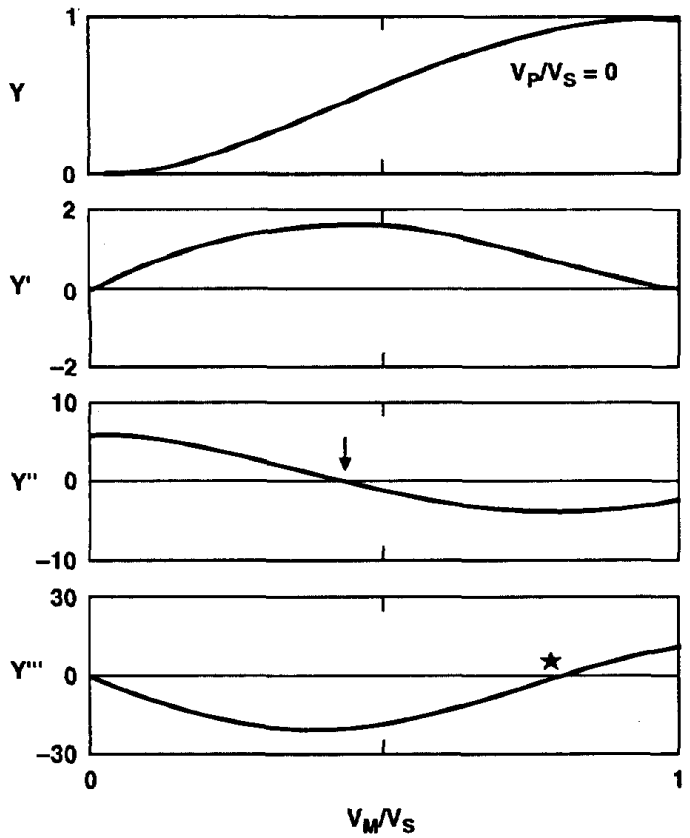

Fig. 7. The transfer curve and its first three derivatives for a directional coupler modulator of electrical length $\theta_{\mathrm{M}}=\pi / 2$ followed by an identical passive section of length $\theta_{P}=\pi / 2$, with normalized bias voltage $V_{P} / V_{S}=0.0$. The proper bias for minimum second harmonic, $V_{M} / V_{S}=0.4394$ is shown by the arrow; the star indicates a possible bias that would make the intermodulation distortion zero, but would result in a large second harmonic.

just touch the to zero line at its maximum, we make both second and third derivatives zero simultaneously, assuring that the second harmonic, third harmonic, and $2 \omega_{1}-\omega_{2}$ outputs are nearly minimized. There will be small remainders at these frequencies produced by the nonzero higher derivatives, which may be canceled by a slight adjustment of the second bias voltage away from $0.73193 V_{S}$ at a single value of modulation drive voltage, just as in the dual MZM previously discussed.

We can apply this same strategy to the three section modulator shown in Fig. 5 in order to find optimum values of $V_{A}$ and $V_{B}$. Fig. 9 shows the transfer function and its first three derivatives for the particular case that $\theta_{M O D}=\pi / 2, \theta_{A}=$ $\theta_{B}=\pi / 4, V_{A} / V_{S}=0.73805$ and $V_{B} / V_{S}=0.77002$. For these values (found by trial and error), second, third, and fourth derivatives are all zero at a modulator bias of $V_{M} / V_{S}=0.509$. Thus, the fourth harmonic will be greatly reduced, the second harmonic will be reduced somewhat from the case of the twosection modulator, and the third harmonic and the $2 \omega_{1}-\omega_{2}$ intermodulation will be of the same order.

It is tempting to speculate that adding further biased sections will add still more degrees of freedom that could be used to set additional derivatives to zero and improve the $2 \omega_{1}-\omega_{2}$ intermodulation. In a study by Sheehy [19] it appears that the fifth derivative may be set to zero, not by adding an additional section, but by moving the second biased section to precede the modulator, and adding phase-shifting lengths between the modulator section and the biased sections. Sheehy also shows that adding further biased electrodes or phase shift sections to the DCM can do no better than this.
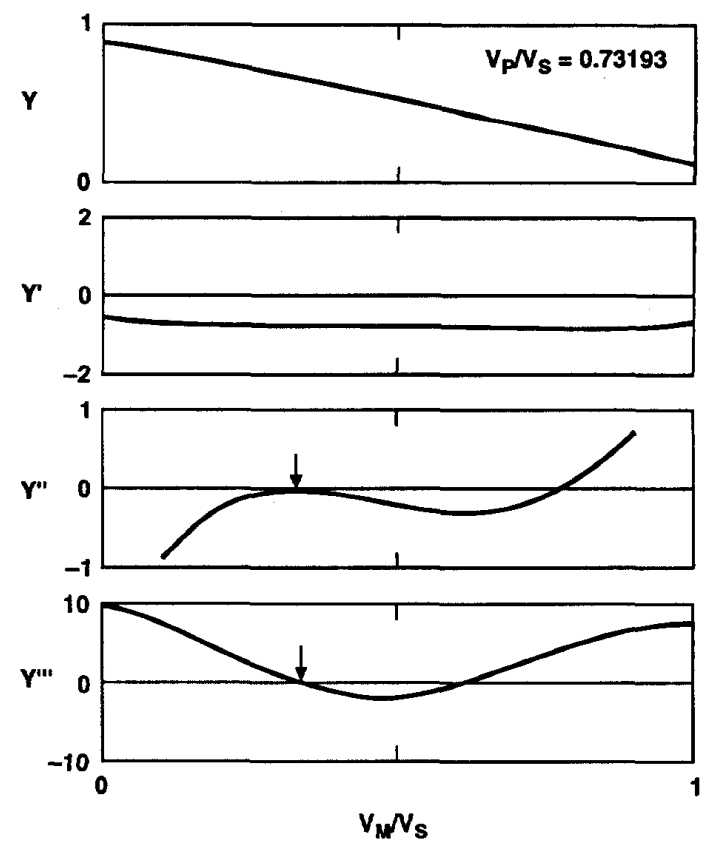

Fig. 8. Same modulator as Fig. 7, but biased to $V_{P} / V_{S}=0.73193$ to simultaneously zero the second and third derivatives.
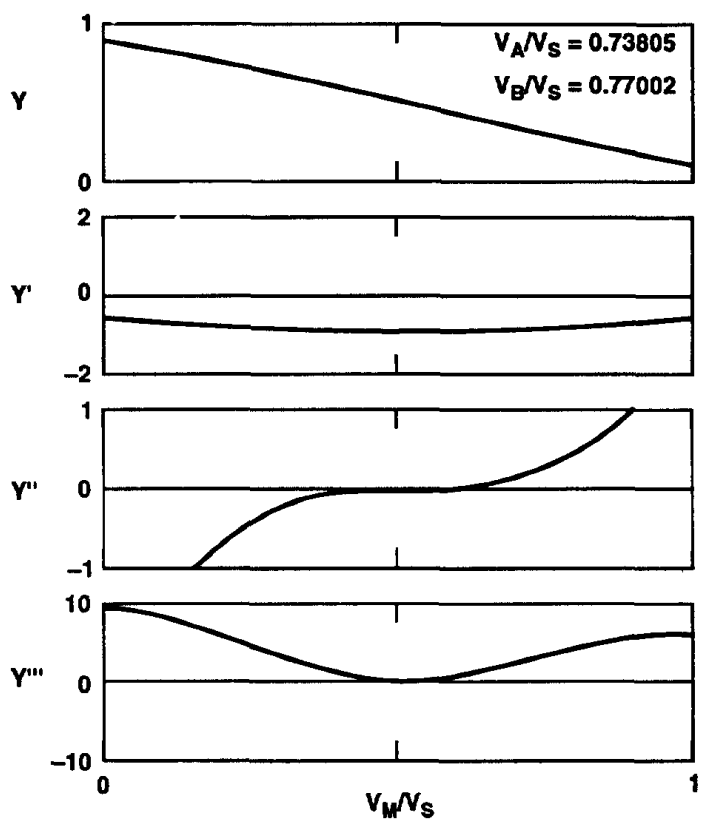

Fig. 9. Transfer function and first three derivatives for the directional coupler modulator of length $\theta_{M}=\pi / 2$ followed by two passive sections of lengths $\theta_{A}=\pi / 4, \theta_{B}=\pi / 4$ as shown in Fig. 5. The biases $V_{A}$ and $V_{B}$ shown were found by trial and error to the maximum dynamic range. The optimum modulator bias is $V_{M} / V_{b}=0.509$.

\section{LINK MODEL}

We now introduce a model for a complete optical link illustrated in Fig. 10, containing a laser source with power $P_{L}[\mathrm{~W}]$, and a relative intensity noise $R I N[\mathrm{~dB} / \mathrm{Hz}]$. The laser feeds a 


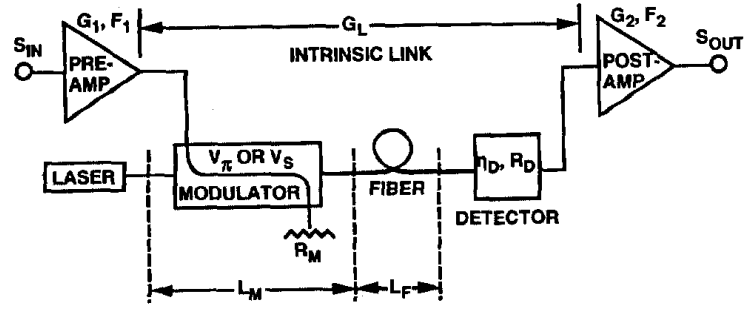

Fig. 10. Schematic of the model that was used in the analysis of high fidelity fiber-optic links. The pre- and post-amplifiers were omitted for most of the calculations.

traveling-wave modulator, which we will describe in terms of its transfer function and its characteristic impedance $R_{M}[\Omega]$. The transfer function will contain a sensitivity characterized by $V_{\pi}$ in the case of MZM's or $V_{S}$ in the case of DCM's, along with bias voltages, optical splits, and other parameters as necessary. The modulator output is attenuated by some loss $L_{o}[\mathrm{~dB}]$, which is the total optical loss in the modulator, the optical fiber, and optical connections $\left(L_{M}+L_{F}\right)$. The optical detector is characterized by its responsivity $\eta[\mathrm{A} / \mathrm{W}]$ and its terminating load $R_{D}[\Omega]$ (which would also be the input impedance of a post amplifier if one were used). The system noise bandwidth is $B W[\mathrm{~Hz}]$.

The modulator is driven by an RF power $P_{\text {in }}[\mathrm{dBm}]$, which consists of two equal amplitude sinusoidal modulations at frequencies 1.0 and $0.9 \mathrm{~Hz}$. This modulation is applied to the transfer function to calculate the output signal. The Fourier components at 1,2 , and $1.1 \mathrm{~Hz}$, corresponding to signal, second harmonic, and intermodulation at $2 \omega_{1}-\omega_{2}$, are calculated by direct numerical integration over the complete period for this signal: $1.0 /(1.0-0.9)=10 \mathrm{sec}$. (The other intermodulation product $2 \omega_{2}-\omega_{1}$ at $0.8 \mathrm{~Hz}$ has the same amplitude as that at $1.1 \mathrm{~Hz}$.) We found direct calculation to be much more satisfactory than taking a numerical FFT, since we are only interested in certain frequency components rather than a complete spectrum; calculating only these components allowed us to program the link model in the user-friendly language MathCAD ${ }^{\circledR}$ on personal computers (486 and Macintosh II). Further details of the calculations are given in the Appendix.

The calculations were made for a consistent set of physical parameters representative (except for the bandwidth) of a typical short fiber-optic link used at microwave modulation frequencies; these are given in Table I. Of the first eight parameters listed in the table, three always occur in the model as the product $H=P_{L} L_{o} \eta_{D}$, which is simply the photodetector current when the modulator transmission is unity; this product is $7 \mathrm{~mA}$ for the values given in the table, and any other values that give the same product will yield the same results. The output Fourier components were calculated for input signal levels from $-160 \mathrm{dBm}$ to $+40 \mathrm{dBm}$. The noise level was calculated over this same range and includes laser $R I N$, shot noise due to the photodiode direct current (assumed to be completely signal-generated; dark current was assumed to be zero), and thermal noise in the input source and output terminating resistors.

The loss $L_{o}$ was taken to be $10 \mathrm{~dB}$, a reasonable value for the fiber, connector, and excess modulator loss (at zero bias) in a short link. For long links, $L_{o}$ will be greater, and an additional noise term accounting for Rayleigh scattering noise should be added.

In addition to plots of the signal, noise, harmonics, and intermodulation as functions of input power, the dynamic range was found by solving numerically for the input RF drive level at which the intermodulation curve intersects the noise level using MathCAD ${ }^{(B)}$ 's root finding routine. The dynamic range was calculated as the difference (in $\mathrm{dB}$ ) between the intermodulation and the signal at this power level. Since the intermodulation curve crossed the noise level multiple times in some cases, the initial guess for the root finding routine was always set to low RF drive levels. The link model program was used in a trial-and-error fashion to adjust the various biases, splits, etc. on the linearized modulators to maximize the dynamic range. The maximum dynamic range as we have defined it above occurs when a subsidiary maximum in intermodulation just "kisses" the noise level, as shown in Fig. 3. Unfortunately, this is just the kind of intersection for which a root-finding routine will have trouble converging. When the root finder failed to converge, a highly magnified plot of the subsidiary maximum and the noise level was generated to decide if the curves kissed or crossed.

The small-signal gain and noise figure were calculated numerically by evaluating the signal at a very small input value, selected typically as $P_{i n}=-100 \mathrm{dBm}$. Since the intrinsic links contain no electronic amplification, their "gains" were actually losses of several tens of $\mathrm{dB}$ and their noise figures were also several tens of $\mathrm{dB}$. Both gain and noise figure is improved by using higher laser power or developing a more sensitive modulator, but it is doubtful that either could be improved sufficiently at microwave frequencies to realize the greater-than-unity gain and good noise figure reported by Cox et al. [14] for low frequency modulators with passive impedance transformations. In almost all applications, electronic amplification would have to be added to a microwave link, and this will introduce an additional source of noise and distortion as discussed later.

\section{Link Performance Results: MACH-ZEHNDER MODULATORS}

For reference we consider first a simple MZM biased properly at $0.5 V_{S}$ to eliminate all even harmonics. Fig. 11 shows the results with the parameters given in Table $\mathrm{I}$. The dynamic range is $109.9 \mathrm{~dB}$ for a $1 \mathrm{~Hz}$ bandwidth, the gain is $-25.2 \mathrm{~dB}$, and the noise figure is $38.0 \mathrm{~dB}$. Since the slope of the intermodulation is closely 3 in the $\log -\log$ plot, it would be easy to define a third order intercept of $3 \mathrm{dBm}$ (output) or $28.2 \mathrm{dBm}$ (input) for this modulator, and use that value to calculate the dynamic range $D Y$ for any other noise level as

$$
D Y=\frac{2}{3}\left(D_{I M}-P_{n o}-B\right)(\mathrm{dBm})
$$

where $D_{I M}$ is the third-order intercept point in $\mathrm{dBm}, P_{n o}$ is the noise power in $\mathrm{dBm}$, and $B$ is the bandwidth in $(\mathrm{dB}) / \mathrm{Hz}$. The third harmonic is about $9.5 \mathrm{~dB}$ below the intermodulation for most of the range.

The results for the dual MZM with the optimum RF drive voltage split of $2.62: 1$ and the "inverse cube" optical split of 


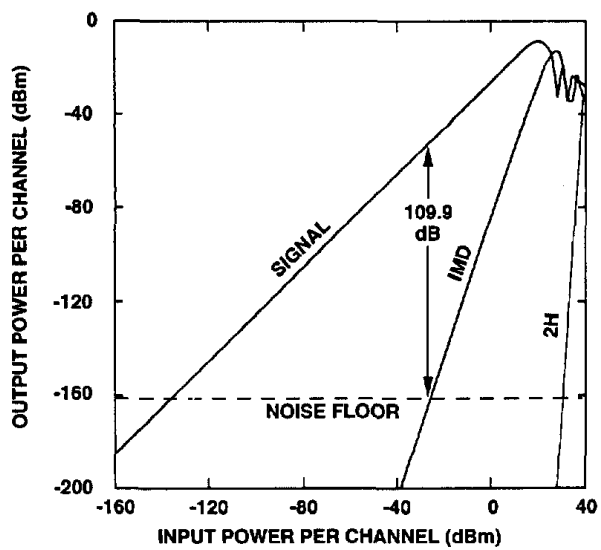

Fig. 11. Signal, intermodulation, and noise for a photonic link (Table I parameters) using a simple Mach-Zehnder modulator biased at $V_{M}=V_{\pi} / 2$. The resulting dynamic range is $109.9 \mathrm{~dB}$. The curve labeled " $2 \mathrm{H}$ " is the component at the second harmonic frequency due to the high-order odd intermodulation products coincidentally at that frequency, since all even order products are identically zero. This curve does depend on the numerical choice of frequencies used.

$1: 17.9847$ were already presented in Fig. 2 for the condition that cancels the cubic term in the intermodulation and results in a simple slope 5 curve. The resulting dynamic range is $126.2 \mathrm{~dB}$ for a $1 \mathrm{~Hz}$ bandwidth, the gain is $-36.0 \mathrm{~dB}$, and the noise figure is $48.8 \mathrm{~dB}$. The gain is lower than the single MZM because there is a partial cancellation of the signal in the process of canceling the intermodulation. And, since the noise level is similar (the noise was split in an uncorrelated fashion between the two detectors) the noise figure is also degraded. (Note also that if the two-polarization scheme were used the detector shot noise would have to be treated slightly differently.)

If either the optical splitting ratio or the RF splitting ratio is adjusted to be slightly off the exact inverse-cube relation, then a small improvement in dynamic range is obtained. Fig. 3 shows the result of making the RF split $2.62: 1$, but the optical split $1: 17.9136$ versus $1: 17.9847$ for the "inverse cubic" relationship, determined by trial and error to produce the maximum dynamic range of $129.7 \mathrm{~dB}$, a $3.5 \mathrm{~dB}$ improvement, with a resulting link gain and a noise figure essentially the same. The dynamic range depends very critically on the RF and optical splitting ratios. Fig. 12 shows the sensitivity of dynamic range to a change in the optical power splitting ratio $O_{1}: O_{2}$ (expressed as the difference $O_{1}-O_{1}$, cUBIC) when the RF voltage is the "inverse cubic" optimum split $2.62: 1$. The sensitivity to change of the RF power ratio $W_{1}: W_{2}$ for the "inverse cubic" optimum optical ratio (expressed as $W_{1}-W_{1}$, CUBIC) is very similar to Fig. 12 . We see that we can gain an improvement in dynamic range above the simple inverse-cubic relation for these splits. But the improvement only comes with very close control of these ratios, a control that likely could be achieved only with active feedback driven by the intermodulation distortion or harmonics from a pilot tone, for example. In fact, to obtain any improvement over a single MZM, not just the "extra" $3.5 \mathrm{~dB}$, active control will likely be necessary.

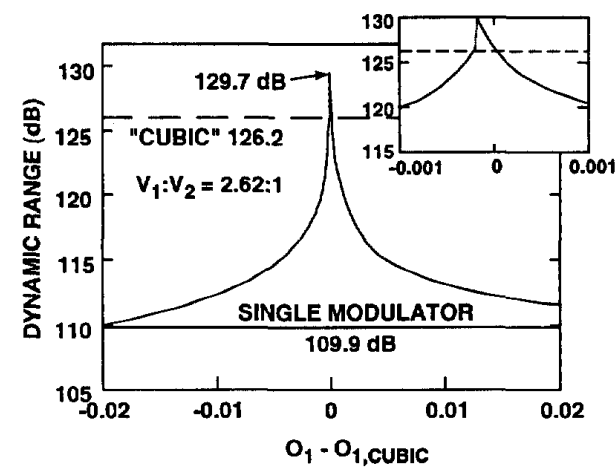

Fig. 12. Tolerance of the dynamic range of the dual-parallel $\mathrm{MZ}$ modulator to changes in the optical power split with the RF split held constant at its "optimum" cubic value. The abscissa is the deviation of $O_{1}$ from its "cubic" value. Total optical power is conserved, so $O_{1}+O_{2}=1$.

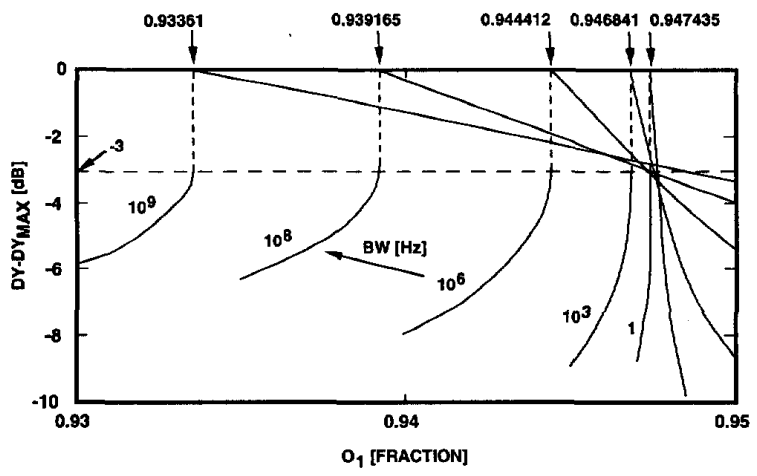

Fig. 13. Change in dynamic range from its maximum value versus the fraction of optical power fed to modulator I for a dual Mach-Zehnder linearization scheme. System noise bandwidth is the parameter from $1 \mathrm{~Hz}-1$ $\mathrm{GHz}$.

The situation is not quite as grim as Fig. 12 implies when we use more realistic values of system noise bandwidth. The intersection of the intermodulation curve with the noise level determines the dynamic range, so that an increased noise level will change the parameters that yield the maximum dynamic range and also the sensitivity to deviations in the parameters. Fig. 12 was calculated for a $1 \mathrm{~Hz}$ bandwidth. Fig. 13 shows the change in dynamic range from its maximum value as a function of the optical fraction used in modulator 1 (the same abscissa as Fig. 12, but shifted by $O_{1, \text { CUBIC) }}$ with system noise bandwidth as a parameter from $1 \mathrm{~Hz}$ to $1 \mathrm{GHz}$. If we measure the "tolerance" to deviations as the width of these curves at some dynamic range degradation, say $-3 \mathrm{~dB}$, then we find the tolerance varies roughly as $(B W)^{1 / 5}$. A perturbation analysis by Hayes [20] that neglects the higher order terms in the intermodulation also predicts a fifth-root variation. Even for $1 \mathrm{GHz}$ bandwidth, Fig. 13 indicates $1 \%$ control will be required on $O_{1}$ to stay within $\pm 1 \mathrm{~dB}$ of a high dynamic range. It is also true that the higher the noise level, the smaller the improvement that can be gained by using a linearized modulator-that is, the ratio of dynamic ranges of the dual $M Z$ to the single MZ. Hayes' perturbation analysis predicts this ratio varies as $(B W)^{-2 / 15}$.

As in the simple MZM, the true second harmonic is identically zero in the dual $\mathrm{MZ}$ because of the symmetry of 


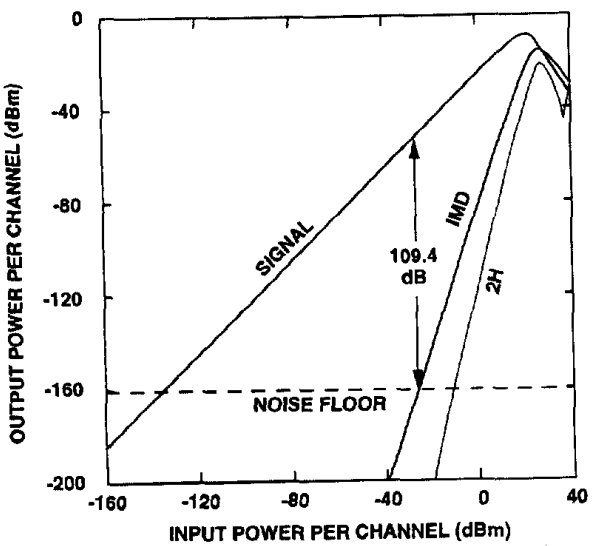

Fig. 14. Output RF signal power and third-order intermodulation power as a function of the input signal power for a fiber-optic link using parameters of Table I and a simple directional coupler modulator. The "standard" bias point of $0.4394 V_{\mathrm{S}}$ was used. In this case the $2 \mathrm{H}$ curve arises form all orders of nonlinearity, including even terms.

the sine-squared transfer function. However, the behavior of the third harmonic in the dual MZM is somewhat more complicated than in the simple MZM. If plotted in Fig. 3, the third harmonic curve would lie below the IMD curve for most of the region to the right of the null in IMD, although by less than the $-9.5 \mathrm{~dB}$ of the simple MZM. For the operating conditions of Fig. 3, a null occurs just to the left of the IMD null, so that the third harmonic is actually greater than the IMD in a very small range of input powers near the value where both third harmonic and IMD enter the noise level. Thus, if third harmonic components fall within the frequency range of interest, the "dynamic range" should be defined by the third harmonic intersection with the noise, rather than the IMD.

It may have occurred to the reader that one might use three identical MZM's and attempt to cancel the $5^{\text {th }}$ order term in the $J_{1}(\theta) J_{2}(\theta)$ Bessel expansion as well as the $3^{\text {rd }}$ order term. This can also be done. The optimum splits for "cubicquintic" cancellation, analogous to the "cubic" condition for the dual MZM are, for RF power, $W_{1}: W_{2}: W_{3}=0.0394$ : $0.3136: 0.6470$ and for optical power, $O_{1}: O_{2}: O_{3}=$ $0.914480: 0.074218: 0.011302$. Using these RF and optical splits, the intermodulation at $2 \omega_{1}-\omega_{2}$ exhibits a smooth slope 7 dependence on the input power. The resulting dynamic range, for the link parameters in Table $\mathbf{I}$, is $132.96 \mathrm{~dB}$, with a small-signal gain of $-41.7 \mathrm{~dB}$ and a noise figure of $54.6 \mathrm{~dB}$. The "second harmonic" at $2 \omega_{1}$ (resulting from coincidental differences between high odd-order terms) is about $2 \mathrm{~dB}$ below the $2 \omega_{1}-\omega_{2}$ intermodulation.

Also analogous to the DMZ, a slightly better dynamic range may be obtained by operating a little off the exact "cubic-quintic" condition, for example, with the same ratio of $W_{1}: W_{2}: W_{3}$ but with $O_{1}: O_{2}: O_{3}=0.914484$ : $0.074218: 0.011298$, we obtain a dynamic range of 134.85 $\mathrm{dB}$. The gain and noise figure are unchanged. The sensitivity of the splits are similar to those shown in Fig. 12. The three MZM scheme is likely only of academic interest; the dual $M Z M$ is hard enough to realize in practice!

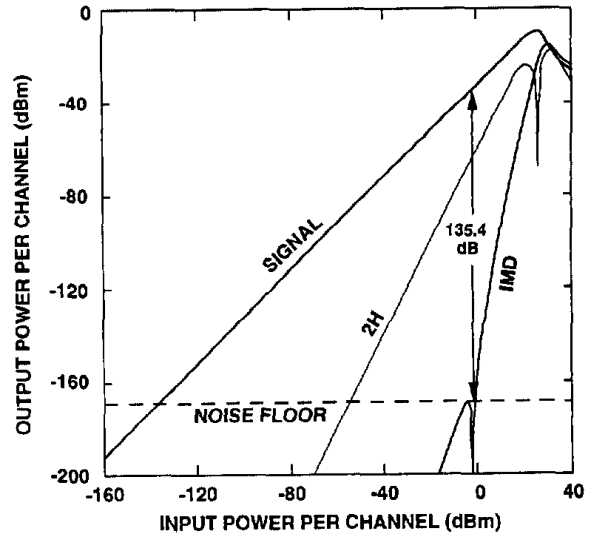

Fig. 15. Output RF signal power, third-order intermodulation power, and second harmonic power as a function of the input signal power for a fiber-optic link (Table I parameters) with a simple directional coupler modulator. The bias point was set to $0.795507 \mathrm{~V}_{\mathrm{s}}$ for maximum dynamic range. The large second harmonic arises from the large quadratic curvature of the transfer function at this bias point.

We conclude that by using a dual MZM and the link parameters given in Table I we can obtain about $20 \mathrm{~dB}$ of improvement in dynamic range at a sacrifice of about $10 \mathrm{~dB}$ in gain and $10 \mathrm{~dB}$ in noise figure, and at a cost of controlling the modulator parameters precisely.

\section{Link PERformance Results: \\ DiRECTIONAL COUPLER MODULATORS}

We now apply the link model calculations to DCM's. Fig. 14 shows the calculated signal, second harmonic, and intermodulation for a simple DCM with the parameters given in Table I. The length of the modulator is chosen to give complete crossover at zero bias. Since there is no bias point that eliminates all even harmonics simultaneously as in the MZM, we have to choose a compromise bias point. For Fig. 14, we have chosen the bias point that minimizes the second harmonic, very near the point that makes the second derivative of the transfer function zero. The residual second harmonic then arises from the nonzero higher even derivatives and shows up as a curve of slope about 4 . The signal, odd harmonics, and $2 \omega_{1}-\omega_{2}$ intermodulation are relatively insensitive to the exact choice of bias in this range. The third harmonic is about $9.5 \mathrm{~dB}$ below the intermodulation, exactly like the MZM. For this link the dynamic range is $109.4 \mathrm{~dB}$, the gain is $-24.8 \mathrm{~dB}$ and the noise figure is $38.0 \mathrm{~dB}$, very close to those values for the simple MZM.

We noted previously (in the caption to Fig. 7) that there is another interesting bias possibility in the simple DCM, at about $0.8 V_{S}$. At this bias the third derivative is zero but the signal is not (the "star" in Fig. 7). And, of course, the second derivative is near its maximum value. Fig. 15 shows the resulting signal, second harmonic and IMD for a bias of $0.795507 V_{S}$, which maximizes the dynamic range to $135.4 \mathrm{~dB}$. The gain is -31.9 $\mathrm{dB}$ and the noise figure is $36.7 \mathrm{~dB}$. The second harmonic would likely be unacceptably large for this link if it fell within the desired pass band $-71 \mathrm{~dB}$ below the signal where the second harmonic equals the noise level. This bias point may be of 
interest for narrow band links where only the IMD falls within the passband.

Next, we consider the DCM followed by a single set of electrodes of the same length as the modulator as described by Lam and Tangonan [3]. Both lengths are chosen to produce complete crossover at zero bias, the same as those used in Figs. 7 and 8 . Adjusting both the modulator bias voltage and the passive section voltage by trial and error to maximize the dynamic range produced the signal, second harmonic, and intermodulation curves shown in Fig. 16. The optimum biases were $0.333719 V_{S}$ for the modulator and $0.73152 V_{S}$ for the dc-biased section when operated in the "cross" state, i.e., the laser input on the $R$-guide and output on the $S$-guide. For these values, the dynamic range was $127.05 \mathrm{~dB}$, the gain was $-31.7 \mathrm{~dB}$, the noise figure was $45.9 \mathrm{~dB}$, and the average light transmission was $64 \%$. It is interesting to consider the output characteristic of the "bar" state, i.e., laser input on the $R$-guide and output on the $R$-guide. The average light transmission on the $R$-guide is $36 \%$, which results in lower shot noise. If the same bias settings are used, however, the signal and IMD will be exactly the same on this arm, which means that the IMD "sidelobe" will now protrude above the noise and yield an inferior dynamic range (about $124 \mathrm{~dB}$ ) compared to the cross arm. However, if a very slight adjustment to the bias is made, e.g., changing $V_{P} / V_{S}$ to 0.731552 from 0.731520 , then the IMD sidelobe falls below the noise and the dynamic range increases to $129.5 \mathrm{~dB}$, the noise figure falls to 42.9 $\mathrm{dB}$, but the gain remains exactly the same. This would be the preferred mode of operation and suggests a general theorem: If the signal and IMD are the same, then the lower the average light transmission the better will be the link dynamic range and noise figure. This theorem is also illustrated by comparing the simple DCM biased at its maximum dynamic range (Fig. 15). The noise figure of the simple DCM is actually better by 6-9 $\mathrm{dB}$, and the dynamic range is about 5-8 dB better than the DCM plus one bias section. This results from a still lower average light transmission of the simple DCM at the $0.79 V_{S}$ bias point, about $7 \%$ compared to 64 or $36 \%$ for the DCM plus secondary section at its optimum bias. The largest contribution to the noise in all three situations is signal shot noise, so minimizing the average light transmission actually helps the noise-dependent link parameters. (Such a strategy was proposed and demonstrated for a simple Mach-Zehnder modulator by Ackerman et al. [21] to increase dynamic range and noise figure by biasing near extinction. Of course, a very large second harmonic results there, too.)

The signal at $2 \omega_{1}$ in the DCM plus one de section from all intermodulation and harmonic terms is greatly improved from the simple DCM biased for maximum dynamic range, Fig. 15, but not as small as that in the simple DCM at its usual bias point, Fig. 14. The second harmonic curve for this modulator could undoubtedly be improved still further if a better "optimization" algorithm had been employed for the second harmonic, as described in the Appendix. Instead, only the value of the second harmonic at the specific input power that made the IMD equal to the noise was used as a measure. While that measure is very low (more than $130 \mathrm{~dB}$ below the signal), the satellite "bump" in $2 H$ at lower inputs was missed.

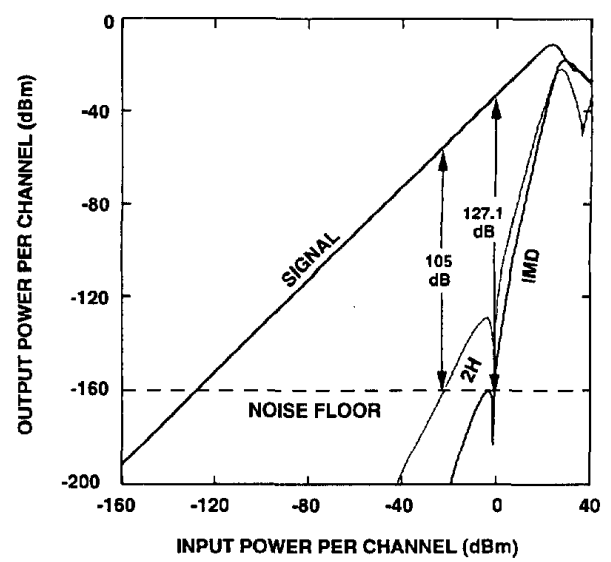

Fig. 16. Output RF signal power, third-order intermodulation power, and second harmonic power as a function of input signal power for a fiber-optic link using the parameters of Table $I$ and a directional coupler modulator with a modulation section of length $\theta_{M}=\pi / 2$ followed by a dc bias section of length $\theta_{P}=\pi / 2$. The bias values $V_{M}=0.340 V_{S}$ and $V_{P}=$ $0.732417 \mathrm{~V} g$ were determined by trial and error to maximize the dynamic range and minimize the second harmonic at the specific input where the IMD equaled the noise level. The second harmonic could be improved with a better optimization algorithm; see the Appendix.

Thus a broadband (greater than an octave) dynamic range for this modulator would be $105 \mathrm{~dB}$, limited by the second harmonic. By relocating the null in the second harmonic using the dynamic range algorithm described in the Appendix we believe the broadband dynamic range could be increased to $127 \mathrm{~dB}$.

Finally, consider the DCM followed by two sets of electrodes, as shown in Fig. 5 and studied by Farwell et al. [4]. The modulator is one transfer length long at zero bias as above, but the two de-biased sections are each half that length. Thus, if the biases applied to the two sections were forced to be equal, this modulator reduces exactly to the previous case. However, allowing the two regions to be biased separately allows a substantially larger linear range, as shown in Fig. 9. Starting with the values scaled from Fig. $9, V_{M}, V_{A}$, and $V_{B}$ were varied by trial-and-error to find the maximum dynamic range and a second harmonic that was everywhere less than the $2 \omega_{1}-\omega_{2}$ IMD. The results for the optimum values are shown in Fig. 17. For this graph, the optimum values were $0.509 V_{S}$ modulator bias, $0.738045 V_{S}$ second section bias, and $0.770017 V_{S}$ third section bias. For these values, the dynamic range is $129.4 \mathrm{~dB}$, the gain is $-30.5 \mathrm{~dB}$, and the noise figure is $43.3 \mathrm{~dB}$, compared to the best $\mathrm{DMZ}$ values of $129.7 \mathrm{~dB}$, $-36.0 \mathrm{~dB}$, and $48.8 \mathrm{~dB}$, respectively. Again, the slightly poorer dynamic range and noise figure compared to the simple DCM at $0.79 V_{S}$ bias (Fig. 15) result from the much higher average light transmission $(49.8 \%)$ and resulting higher shot noise. Since the light transmission is so close to $50 \%$, both "cross" and "bar" state operation will be the same.

The second harmonic lies significantly below the IMD, and exhibits two nulls, as shown in Fig. 17. The third harmonic curve (not shown) lies below the IMD curve by about $5 \mathrm{~dB}$ over almost the entire range to the right of the IMD null, but remains slightly above the noise level at the IMD null, since 


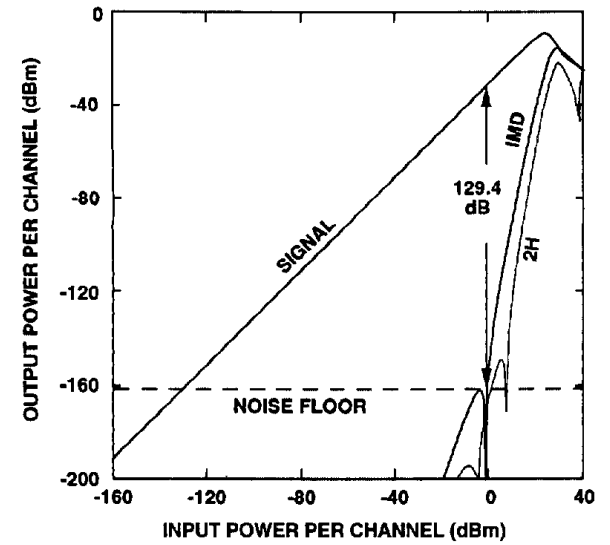

Fig. 17. Output signal, intermodulation and second harmonic for a direc tional coupler modulator with two passive bias sections each one-half the length of the modulator. Bias points of $V_{h}=0.509 \mathrm{~V}_{\mathrm{S}}, V_{A}=0.738045 \mathrm{~V}_{S}$, and $V_{B}=0.770017 T^{5}$ were found to be optimum by trial and error.

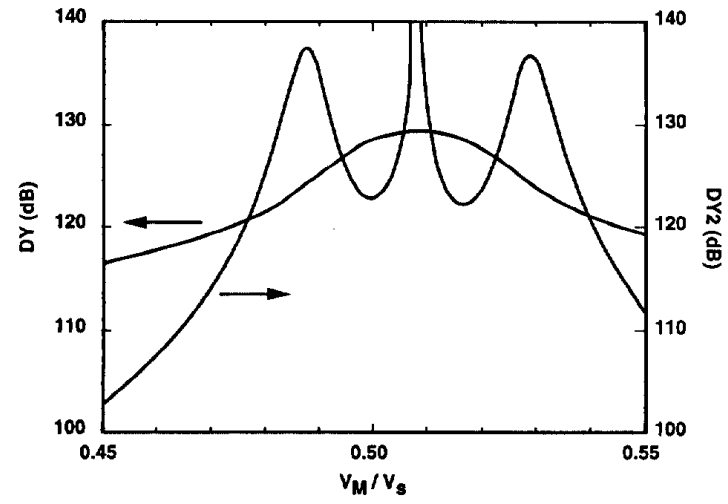

Fig. 18. Dynamic range (left scale) and signal-to-second harmonic ratio at the input power where the $2 \omega_{1}-\omega_{2}$ intermodulation intersects the noise level (right scale) as a function of modulator bias point for a photonic link with parameters given in Table $I$ and a linearized directional coupler modulator with two passive bias sections at $V_{A}=0.770017 \mathrm{~V}_{\mathrm{S}}$.

the third harmonic null lies just to the left of the IMD null. This is similar to the situation for the dual MZM.

It is important to consider the sensitivity of the above results to the errors in the three bias settings, analogous to errors in optical and RF power splits for the dual MZM. Fig. 18 shows the sensitivity of the IMD and a measure of the second harmonic (see Appendix for definition) and IMD to the modulator bias setting; not surprisingly, the results are relatively insensitive to this bias, since what we have set out to do is make a linear curve for the modulator transfer function. Errors of a few percent in setting the modulator section bias would not change the modulator performance significantly. By contrast, Fig. 19 shows the sensitivity of the second harmonic and IMD to variation in the second section bias. Here, changes of $\pm 0.01 \%$ would reduce the dynamic range by $5 \mathrm{~dB}$. Of course, Fig. 19 is for a $1 \mathrm{~Hz}$ bandwidth, and we expect a similar decrease in sensitivity by $B W^{1 / 5}$. Thus we would expect $\pm 0.16 \%$ for $1 \mathrm{MHz}$ and $\pm 0.6 \%$ for $1 \mathrm{GHz}$ bandwidths,

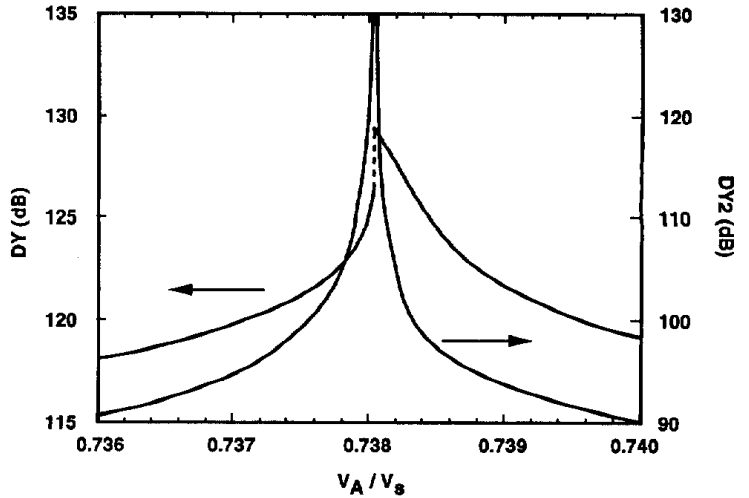

Fig. 19. Dynamic range (left scale) and second harmonic (right scale) as a function of first section bias voltage $V_{A}$ with $V_{A}$ at $0.509 V_{S}$ and $V_{B}=$ $0.770017 \mathrm{Vs}$

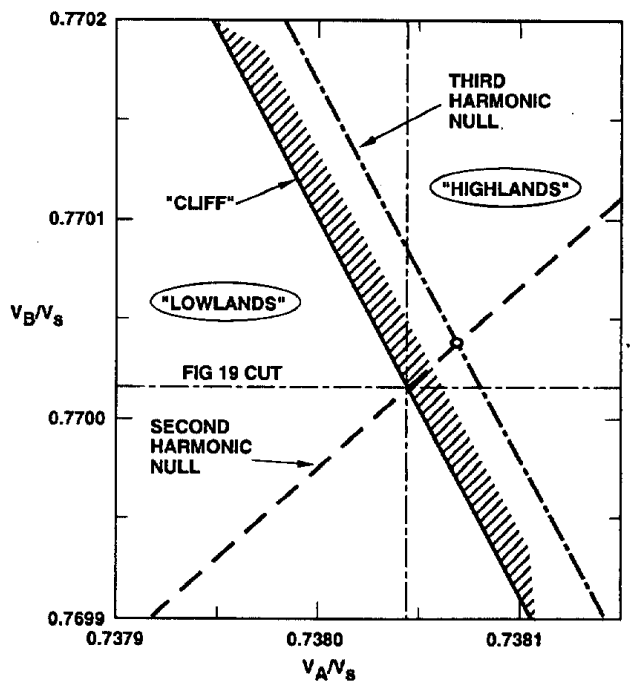

Fig. 20. Locus of the maximum dynamic range ("cliff") for the DCM of Figs. 17-19. Also shown are the minimum second and third harmonic null loci in the $V_{A}-V_{B}$ plane for an applied pilot tone of $-4 \mathrm{dBm}$.

although we have not made the calculations. The variation with third section bias voltage $V_{B} / V_{S}$ is very similar to Fig. 19 .

Fig. 20 plots the position of the "cliff" or discontinuity in dynamic range as a function of the two biases. Pairs of biases along the "cliff" line will all produce dynamic ranges of the order of $129.4 \mathrm{~dB}$, while east-west motion will produce the curve of Fig. 19. Also shown in this figure are the loci of biases that will produce a null in second and third harmonics for an applied pilot tone power of $-4 \mathrm{dBm}$. At the intersection of the second and third harmonic null loci (open circle), the dynamic range is $129.0 \mathrm{~dB}$, only $0.4 \mathrm{~dB}$ less than the optimum value of the edge of the "cliff." Thus only a little dynamic range would be lost in an active bias stabilization scheme based on nulling the second and third harmonics of a pilot tone, as suggested by Hayes [15]. The pilot tone amplitude also needs to be stabilized since the third harmonic curve moves relative to the "cliff" as the amplitude varies. A pilot tone of $-6 \mathrm{dBm}$ moves the third harmonic null curve farther to the right, thus 
selecting an operating point still in the "highlands," but with a smaller dynamic range; a pilot tone of $0 \mathrm{dBm}$ moves the third harmonic null curve to the left, into the "lowlands," selecting an operating point with several $\mathrm{dB}$ smaller dynamic range. Of course, this stability requirement should be relaxed with system noise bandwidths greater than $1 \mathrm{~Hz}$.

\section{Link Performance with A Preamplifier}

We have addressed the effects of electronic amplifiers on optical link performance in a previous paper [9], an extension of still older work for cascaded electronic amplifiers [16]. Clearly, the intrinsic optical links (i.e., without electronic preor post-amplification) described above will require the addition of electronic amplifiers to produce acceptable overall link gain and noise figure. And the distortions produced in such amplifiers will add to those produced in the modulator. We also modified our numerical programs to include preamplifiers with given small-signal gain, noise figure, third-order intercept (TOI), and second-order intercept (SOI). Gain saturation in the preamplifier is ignored. The IMD and $2 H$ outputs of the preamplifier are calculated and then passed through the modulator using the numerically calculated slope of the transfer curve. The preamplifier distortion and modulator distortion are added in quadrature at the photodetector, since they arise from physically independent sources and are thus uncorrelated.

We can illustrate the effect of adding a preamplifier to a linearized DCM, one with two added bias sections as described in Figs. 17-20. We chose a range of preamplifier parameters that encompass those of the best obtainable microwave amplifiers, but also include values that are better than realizable at the present. Fig. 21 shows how the dynamic range and noise figure of the intrinsic link are changed as a function of preamplifier gain from $0-50 \mathrm{~dB}$. The preamplifier noise figure is $3 \mathrm{~dB}$ and its third-order intercept varies from 40 $\mathrm{dBm}$ (off-the-shelf item) to $60 \mathrm{dBm}$. As expected from the Friis formula [17], for amplifier gains of the order of the link loss, the overall noise figure approaches the preamplifier noise figure. The link dynamic range, however, depends little on the preamplifier gain up to $30-40 \mathrm{~dB}$, but depends critically on the TOI of the preamplifier. A preamplifier TOI greater than 60 $\mathrm{dBm}$ would be required to keep the link dynamic range from degrading by $3 \mathrm{~dB}$. At gains in the $40-50 \mathrm{~dB}$ range, further degradation in dynamic range takes place as the modulator begins to contribute to the distortion. The conclusion here, as it was in [9], is that it makes no sense to use a highly linearized modulator unless the driving preamplifier has a high TOI.

Similarly, distortion introduced by the nonideal behavior of the optical detector could be included in the overall link behavior. The high optical powers encountered in short microwave links likely will produce such nonideal behavior. Both the very small area photodetectors that are required for microwave output and the dependence of the link gain and noise figure on the laser power yield designs with high optical power densities on the photodetector. Hayes and Persechini [18] have measured the distortion produced in typical microwave photodetectors, and it is significant enough that degrades the link dynamic range even further.

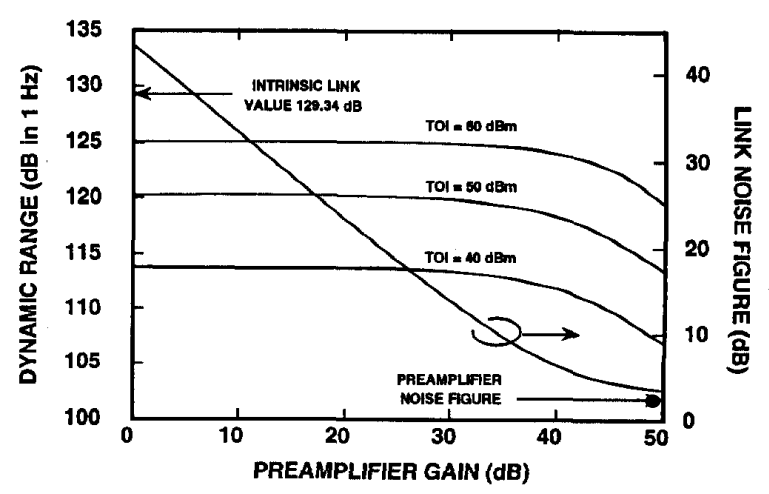

Fig. 21. Dynamic range and noise figure for an optical link with a preamplifier as a function of preamplifier gain with preamplifier third-order intercept as a parameter. The modulator is the DCM with two added bias regions, Figs. 5 and 17-20, and the preamplifier noise figure is $3 \mathrm{~dB}$.

\section{CONCLUSION}

We have developed a simple link model that calculates harmonic and intermodulation distortion by Fourier-analyzing the link output when a two-tone input signal is applied. We have applied the model to selected linearized modulator schemes, particularly the dual and triple Mach-Zehnder, and directional coupler modulators with zero, one or two additional dc-biased regions to enhance linearity. We find that the harmonics and intermodulation produced no longer exhibit a simple constantexponent power law behavior with the input signal, and we propose a new, unambiguous definition of dynamic range to cope with this added complexity. For a sample set of parameters, we calculate that improvements of about $20 \mathrm{~dB}$ in dynamic range are obtainable, at a sacrifice of $10 \mathrm{~dB}$ in gain and noise figure, but that the modulator parameters must be tightly controlled to realize such an improvement. Table II summarizes the link performances for a $1 \mathrm{~Hz}$ noise bandwidth. We also demonstrate that the addition of low noise electronic amplifiers with even the best obtainable third-order intercepts will significantly degrade the dynamic range.

\section{APPENDIX}

\section{A. Link Model Calculations}

The MathCAD ${ }^{\circledR}$ program inputs the link parameters listed in Table I plus the parameters that enter into the particular modulator transfer function $Y(V)$, described later in this appendix. The transfer function gives the fractional optical transmission through the modulator when a normalized voltage $V / V_{S}$ or $V / V_{\pi}$ ) is applied ( $V_{S}$ is the DCM transfer voltage and $V_{\pi}$ is the MZM half-wave voltage). The independent variable used in the link model is the input power $P_{i n}(\mathrm{dBm})$. This value in $\mathrm{dBm}$ is converted to power in Watts, $S_{i n}\left(P_{i n)}\right.$.

The normalized voltage applied to the modulator is then given by

$$
V\left(t, P_{i n}\right)=\frac{V_{M}}{V_{s, \pi}}+\frac{\sqrt{2 R_{M} S_{i n}\left(P_{i n}\right)}}{V_{s, \pi}} \cdot x(t)
$$


TABLE II

Performance of Fiber-Optic Links with Parameters of Table I

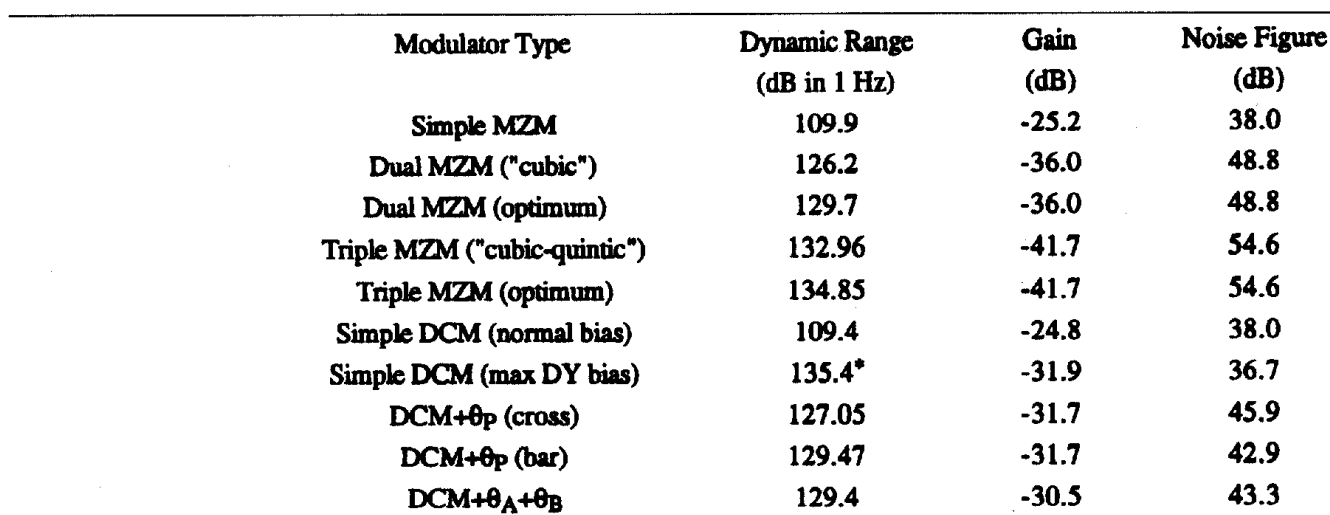

"Dynamic range based on second harmonic only is $82 \mathrm{~dB}$ (in $1 \mathrm{HZ}$ )

where $V_{M}$ is the bias voltage, $R_{M}$ is the modulator characteristic impedance, and $x(t)$ is the (dimensionless) applied two-tone signal at frequencies $a$ and $b$

$$
x(t)=\sin (2 \pi a t)+\sin (2 \pi b t) .
$$

In all calculations $a$ and $b$ are taken as 1 and $0.9 \mathrm{~Hz}$, respectively, so the intermodulation products $2 a-b$ and $2 b-a$ occur at 1.1 and $0.8 \mathrm{~Hz}$, respectively. We calculate only the former. Second and third harmonics are taken as $2 a=2 \mathrm{~Hz}$ and $3 a=3 \mathrm{~Hz}$. The results are, of course, independent of the absolute frequency value except for the chance coincidence in high order intermodulation products that happen to fall at critical frequencies, e.g., $11 a-10 b=2 \mathrm{~Hz}$.

The waveform as distorted by the modulator transmission function $Y(V)$ is then obtained from $Y\left[V\left(t, P_{i n}\right)\right]$. To avoid "saturating" the Fourier transform integrals numerically, the dc component of transmission, $Y\left[V_{M}^{J} / V_{S, \pi}\right]$, is subtracted from $Y$ to yield the time-varying component $\hat{Y}\left(t, P_{i n}\right)$.

The Fourier components of this RF waveform are then calculated directly using MathCAD ${ }^{\circledR}$ 's numerical integration routine. The integrands are scaled inversely with the input voltage by multiplying by $\left[S_{i n}\left(P_{i n}\right)\right]^{-1 / 2}$, again, to keep the integrands from becoming too small and suffering from roundoff errors. In some cases additional fixed scaling factors were used over different ranges of $P_{i n}$ to speed computation time in evaluating these integrals ${ }^{3}$

In addition, it is necessary to calculate both in-phase and quadrature components of each quantity to account for possible phase shifts introduced by the transfer function. Thus, the

\footnotetext{
${ }^{3}$ It would likely be better to use different scale factors for IMD and second harmonic, say $S_{i n}^{-3 / 2}$ for IMD and $S_{i n}^{-1}$ for second harmonic. However, in the linearized modulators, the dependence on input power is not so simple, and it was not immediately obvious how to choose the proper integrand scaling in advance.
}

signal component is given by

$$
\begin{aligned}
\operatorname{SIG}\left(P_{i n}\right)= & {\left[S_{i n}\left(P_{i n}\right)\right]^{1 / 2} } \\
& \cdot\left\{\left[\frac{2}{T} \int_{0}^{T}\left[S_{i n}\left(P_{i n}\right)\right]^{-1 / 2}\right.\right. \\
& \left.\cdot \hat{Y}\left(t, P_{i n}\right) \sin (2 \pi a t) d t\right]^{2} \\
& +\left[\frac{2}{T} \int_{0}^{T}\left[S_{i n}\left(P_{i n}\right)\right]^{-1 / 2}\right. \\
& \left.\left.\cdot \hat{Y}\left(t, P_{i n}\right) \cos (2 \pi a t) d t\right]^{2}\right\}^{1 / 2}
\end{aligned}
$$

The integrals are taken over an exact period $T$ of the input waveform, $T=1 /(b-a)=10 \mathrm{sec}$. for $a=1 \mathrm{~Hz}$ and $b=0.9 \mathrm{~Hz}$. The third-order intermodulation $\operatorname{IMD}\left(P_{\text {in }}\right)$ is calculated in the same fashion by substituting $2 a-b$ for $a$ in equation (A-5), and the second and third harmonic $2 H\left(P_{i n}\right)$ and $3 H\left(P_{i n}\right)$ by substituting $(2 a)$ and $(3 a)$, respectively. These quantities are actually the Fourier components of the time-varying modulator transmission. They are converted to detector current by multiplying by the unity transmission detector current $H=P_{L} L_{o} \eta_{D}$, where $P_{L}$ is the laser power in Watts, $L_{o}$ is the total optical insertion loss ratio from the laser to the detector, and $\eta_{D}$ is the detector responsivity (A/W). Signal, intermodulation, and harmonics are further converted to average RF powers in $\mathrm{dBm}$ at the output resistor $R_{D}$ of the intrinsic link: $P S I G \mathrm{dBm}\left(P_{i n}\right), P \operatorname{IMD} \mathrm{dBm}\left(P_{i n}\right), P 2 H$ $\mathrm{dBm}\left(P_{i n}\right)$, and $P 3 H \mathrm{dBm}\left(P_{i n}\right)$.

The small signal gain of the link, $G$, is obtained by evaluating the output at a very small value of input power, $P_{\text {in }}=-100 \mathrm{dBm}$. For the intrinsic link, $G$ is typically much less than unity, say $10^{-3}-10^{-4}$. The noise output power [W/ $\mathrm{Hz}^{-1}$ ] in $R_{D}$ is given by

$$
N=G k T+R I N \cdot I_{D C}^{2} R_{D}+2 e I_{D C} R_{D}+k T
$$


where $R I N$ is the relative intensity noise of the laser (expressed as a ratio), and $I_{D C}$ is the average photocurrent $H \cdot Y\left[V_{M} / V_{S}, \pi\right]$. Note that both the thermal noise $G k T$ from the modulator driving source (assumed to be matched to $R_{M}$ ) and $k T$ from the detector terminating resistor $R_{D}$ are included even though the former is negligible compared to the latter for typical $G$ 's less than unity. The noise figure is then $F \mathrm{dBm}=N \mathrm{dBm}-[G k T]_{\mathrm{dBm}}$. If a post amplifier is added, the $k T$ from $R_{D}$ should be deleted before calculating the link noise figure so that it may be correctly cascaded with the post amplifier via the Friis formula [18].

The link dynamic range is calculated by first finding $X_{i n}$, the particular value of input power $P_{i n}$ that makes the intermodulation equal to the noise. MathCAD ${ }^{\circledR}$ 's rootfinder is used to solve the equation $P I M \mathrm{dBm}\left(P_{i n}\right)-N \mathrm{dBm}=0$ for the root $X_{i n}$. The dynamic range in $\mathrm{dB}$ is then $D Y=P S I G \mathrm{dBm}$ $\left(X_{i n}\right)-N \mathrm{dBm}$. When MathCAD's root-finding program has difficulty converging, which usually happens when the solution is near the "kissing" point of a subsidiary maximum with the noise level, then a program that plots both intermodulation and noise is used to find the desired solution by successive trials.

A measure of the signal-to-second harmonic ratio, $D Y 2$, is found by evaluating this ratio at $X_{i n} ; D Y 2=P S I G \mathrm{dBm}$ $\left(X_{i n}\right)-P 2 H \mathrm{dBm}\left(X_{i n}\right)$. Depending on the complexity of the $P I M \mathrm{dBm}$ and $P 2 H \mathrm{dBm}$ curves, $D Y 2$ can be used to estimate whether the second harmonic is significant or not compared to the IMD. But note that the measure $D Y 2$ is not a true dynamic range for the second harmonic. Such a true value would be found by solving the equation $\mathrm{P} 2 \mathrm{H} \mathrm{dBm}\left(P_{i n}\right)-N$ $\mathrm{dBm}=0$ for the lowest-valued root in input power, $X 2_{i n}$, and then evaluating True $D Y 2=P S I G \mathrm{dBm}\left(X 2_{i n}\right)-P 2 H$ $\mathrm{dBm}\left(X 2_{i n}\right)$. The true broadband dynamic range would then be determined by the smaller of $D Y$ and True $D Y 2$ (assuming that the third harmonic is always less than IMD). We did not use this procedure in optimizing the modulator parameters, and Fig. 16 is an unfortunate less-than-optimum result as a consequence of looking only at $D Y 2$ and not True $D Y 2$.

\section{B. Transfer Functions}

For the Mach-Zehnder modulator, the transfer function is simply

$$
Y(V)=\left[\sin \left(\frac{\pi V}{2}\right)\right]^{2}
$$

where $V$ is the voltage normalized to $V_{\pi}$, as in (A-2).

For the dual MZM, the additional parameters required are the lossless RF power splitting ratio $F:(1-F)$, the lossless optical power splitting ratio $f:(1-f)$, and the two modulator bias voltages ${ }^{1} V_{M}$ and ${ }^{2} V_{M}$ (denoting the two modulators by pre-superscript "l" and "2"). The RF and optical powers fed to the two modulators are thus

$$
\begin{aligned}
{ }^{1} S_{i n}\left(P_{i n}\right) & =F S_{i n}\left(P_{i n}\right) \\
{ }^{2} S_{i n}\left(P_{i n}\right) & =(1-F) S_{i n}\left(P_{i n}\right) \\
{ }^{1} H & =f H \\
{ }^{2} H & =(1-f) H .
\end{aligned}
$$

The transfer function is thus

$$
\begin{aligned}
Y(V)= & f \sin ^{2}\left\{\pi\left[\frac{{ }^{1} V_{M}}{V_{\pi}}+\sqrt{F} \cdot \frac{\sqrt{2 R_{M} S_{i n}\left(P_{i n}\right)}}{V_{\pi}}\right]\right\} \\
& +(1-f) \sin ^{2}\left\{\pi \left[\frac{{ }^{2} V_{M}}{V_{\pi}}+\sqrt{(1-F)}\right.\right. \\
& \left.\left.\cdot \frac{\sqrt{2 R_{M} S_{i n}\left(P_{i n}\right)}}{V_{\pi}}\right]\right\} .
\end{aligned}
$$

To obtain cancellation of intermodulation and odd harmonics, the two modulators must be biased to opposite slopes. In all calculations, we took ${ }^{1} V_{M} / V_{\pi}=1 / 2$ and ${ }^{2} V_{M} / V_{\pi}=-1 / 2$.

The simple directional coupler modulator is usually defined by its physical length $l$, coupling coefficient $\kappa$, and the difference in the propagation coefficients of the two arms, $\Delta \beta$. We chose to express these as the coupling angle $\theta_{M}=\kappa l$ and the transfer voltage $V_{S}$, that is, the voltage applied to the electrodes that causes the full output to switch from one arm to the other arm when $\theta_{M}$ is $n \pi / 2$ ( $n$ an integer). Thus a DCM with $\theta_{M}=\pi / 2$ and an optical input into arm $R$ only would have an optical output only from arm $S$ at zero applied voltage, and output only from arm $R$ with $V_{S}$ applied. The transfer function for arm $R$ to arm $S$ is then

$$
Y_{R S}(V)=\frac{\left[\sin \left(\theta_{M} \cdot \sqrt{1+3 V^{2}}\right)\right]^{2}}{1+3 V^{2}}
$$

where $V$ is the applied voltage normalized to $V_{S}$. We have assumed the two arms are identical, so that $\Delta \beta=0$ with zero applied voltage; the formula is easily modified to include a static $\Delta \beta$. The transfer function from input to output in arm $R$ is $Y_{R R}(V)=1-Y_{R S}(V)$ and $Y_{S R}=Y_{R S}, Y_{S S}=Y_{R R}$.

The transfer function for the DCM with additional sets of electrodes is somewhat more complicated to define. We must work with optical amplitudes rather than powers, since we must keep track of the modulation phase as the signals pass from section to section. It is convenient to consider the transfer matrix from input arms $R$ and $S$ to output arms $R$ and $S$

$$
M=\left(\begin{array}{cc}
M_{R R} & -j M_{R S} \\
-j M_{S R}^{*} & M_{S S}^{*}
\end{array}\right) .
$$

Note that there are only two independent elements

$$
\begin{aligned}
M_{R R}= & M_{S S} \\
= & \cos \left[\theta_{M} \sqrt{1+3 V^{2}}\right] \\
& +j \frac{\sqrt{3} V \sin \left[\theta_{M} \sqrt{1+3 V^{2}}\right]}{\sqrt{1+3 V^{2}}}
\end{aligned}
$$

and

$$
\begin{aligned}
M_{R S} & =M_{S R} \\
& =\frac{\sin \left[\theta_{M} \sqrt{1+3 V^{2}}\right]}{\sqrt{1+3 V^{2}}} .
\end{aligned}
$$

We note that $Y_{R S}(V)$ is simply $M_{R S} M_{R S}^{*}$ in (A-28).

If additional passive sections of length $\theta_{A}$ and $\theta_{B}$ are added, with normalized bias voltage $V_{A}$ and $V_{B}$, then they can be described by additional matrices $A, B$ with elements $A_{i j}, B_{i j}$, obtained by appropriate substitutions in (A-30)-(A-32). The overall amplitude transmission matrix $\boldsymbol{T}$ may be obtained by matrix multiplication, $\boldsymbol{T}=\boldsymbol{B} \boldsymbol{A M}$, with elements $T_{i j}$. The 
crossover amplitude is found to be

$$
\begin{aligned}
T_{R S}(V)= & -j\left\{B_{R S}^{*}\left[A_{R R} M_{R R}(V)-A_{R S} M_{R S}^{*}(V)\right]\right. \\
& \left.+B_{R R}^{*}\left[A_{R S}^{*} M_{R R}(V)+A_{R R}^{*} M_{R S}^{*}(V)\right]\right\}
\end{aligned}
$$

and the desired optical intensity transfer functions is then $Y_{R S}(V)=T_{R S}(V) T_{R S}^{*}(V)$. If only one passive section is used, set $V_{A}=V_{B}=V_{P}$ and $\theta_{A}=\theta_{B}=\theta_{P} / 2$. And, of course, the simple DCM is obtained with $\theta_{A}=\theta_{B}=0$, albeit with a longer-running program.

\section{ACKNOWLEDGMENT}

The authors would like to thank R. H. Buckley, R. R. Hayes, J. F. Lam, and G. L. Tangonan of Hughes Research Laboratories; N. P. Bernstein of the Air Force Rome Laboratory; B.M. Hendrickson of the Air Force Rome Laboratory and ARPA; B. Hui of ARPA; G. E. Betts and C. H. Cox of Lincoln Laboratories; F.T. Sheehy of Caltech (now with McKinsey and Co.), and R. A. Becker of IOCC for many helpful discussions and comments.

\section{REFERENCES}

[1] G. E. Betts, "Linearized modulator for suboctave-bandpass optical analog links," IEEE Trans. Microwave Theory Tech., vol. 42, no. 12, pp. 2642-2649, Dec. 1994.

[2] S. K. Korotky and R. M. Ridder, "Dual parallel modulation schemes for low-distortion analog optical transmission," IEEE J. Select. Areas Commun., vol. 8, no. 7, pp. 1377-1381, Sept. 1990.

[3] J. F. Lam and G. L. Tangonan, "Optical modulation system with enhanced linearization properties," IEEE Photon. Technol. Lett., vol. 3, no. 12, pp. 1102-1104, Dec. 1991

[4] M. L. Farwell, Z. Q. Lin, E. Wooten, and W. S. C. Chang, "An electrooptic intensity modulator with improved linearity," IEEE Photon. Technol Lett. vol 3, no. 9, pp. 792-795. Sept 1991.

[5] J. H. Schaffner, J. F. Lam, C. J. Gaeta, G. L. Tangonan, R. L. Joyce, M. L. Farwell, and W. S. C. Chang, "Spur tree dynamic range measurements of a fiber optic link with traveling wave linearized directional coupler modulators," IEEE Photon. Technol. Lett. vol. 6, no. 2, pp. 273-275. Feb. 1994.

[6] J. L. Brooks, G. S. Maurer, and R. A. Becker, "Implementation and evaluation of a dual parallel linearization system for AM-SCM video transmission," J. Lightwave Technol., vol. 11, no. 1, pp. 34-41, Jan. 1993.

[7] Y. Wang-Boulic, "A linearized optical modulator for reducing thirdorder intermodulation distortion," J. Lightwave Technol., vol. 10, no. 8, pp. 1066-1070. Aug. 1992.

[8] P.-L. Liu, B. J. Li, and Y. S. Trisno, "In search of a linear electrooptic amplitude modulator," IEEE Photon. Technol. Lett. vol. 3, no. 2, pp. 144-146, Feb. 1991

[9] 3. H. Schaffner and W. B. Bridges, "Intermodulation distortion in high dynamic range microwave fiber optic links with linearized modulators," J. Lightwave Technol., vol. 11, no. 1, pp. 3-6, Jan. 1993.

[10] L. M. Johnson and H, V. Roussell, "Reduction of intermodulation distortion in interferometric optical modulators," Opt. Lett., vol. 13, no. 10. pp. 928-930. Oct. 1988.

[11] R. V. Schmidt, "Integrated optics switches and modulators," in Integrated Optics: Physics and Applications, S. Martellucci and A. N. Chester, Eds. New York: Plenum, 1981, pp. 181-210.

[12] T. R. Halemane and S. K. Korotky, "Distortion characteristics of optical directional coupler modulators," IEEE Trans. Microwave Theory Tech., vol. 38, no. 5, pp. 669-673, May 1990

[13] For example, as done by Lam and Tangonan in [3] using Mathematica.

[14] C. H. Cox III G. E Betts, and L M Johnson, "An analytic and experimental comparison of direct and external modulation in analog fiber-optic links," IEEE Trans. Microwave Theory Tech., vol. 38, no. 5 , pp. 501-509, May 1990.

[15] R. R. Hayes, private communication.

[16] N. G. Kanaglekar, R. E. McIntosh, and W. E. Bryant, "Analysis of two-tone, third-order distortion in cascaded two-ports," IEEE Trans. Microwave Theory Tech., vol. 36, no. 4, pp. 701-705, Apr. 1988.

[17] H. T. Friis, "Noise figures of radio receivers," Proc. IRE, vol. 32, pp. 4 J9-422, July 1944
[18] R. R. Hayes and D. L. Persechini, "Nonlinearity of PIN photodetectors," IEEE Photon. Technol. Lett., vol. 5, no. 1, pp. 70-72, Jan. 1993.

[19] F. T. Sheehy, "Antenna-coupled mm-wave electro-optic modulators and linearized electro-optic modulators," Ph.D. dissertation, California Institute of Technology, Pasadena, CA, June 1993.

[20] R. R. Hayes, Hughes Research Laboratories, unpublished memo.

1211 E. Ackerman, D. Kasemset, and S. Wanuga, "An external modulation Lband link with a $117 \mathrm{~dB}$ spurious signal-free dynamic range," Microwave $J$. vol. 34, pp. 158-164, Sept. 1991.

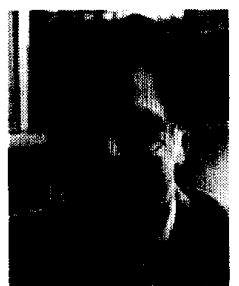

William B. Bridges (S' 53-M'61-F'70) was born in Inglewood, CA, in 1934. He received the B.S., M.S. and $\mathrm{Ph} . \mathrm{D}$. degrees in electrical engineering from the University of California, Berkeley, in 1956, 1957. and 1962 , respectively.

$\mathrm{He}$ was an Associate in Electrical Engineering at the same university from 1957-1959, teaching courses in communication and circuits. His graduate research dealt with noise in microwave tubes and elecron-stream instabilities (which later became the basis of the Vircator). Summer jobs at RCA and Varian provided stimulating experience with microwave radar systems, ammonia beam masers, and the early development of the ion vacuum pump. He joined the Hughes Research Laboratories in 1960 as a member of the Technical Staff and was a Senior Scientist from 1968-1977, with a brief tour as Manager of the Laser Department in 1969-1970. His research at Hughes involved gas lasers of all types and their application to optical communication, radar, and imaging systems. He is the discoverer of laser oscillation in noble gas ions and spent several years on the engineering development of practical highpower visible and ultraviolet ion lasers for military applications. He joined the faculty of the California Institute of Technology in 1977 as Professor of Electrical Engineering and Applied Physics. He served as Executive Officer for Electrical Engineering from 1979-1981. In 1983, he was appointed Carl F. Braun Professor of Engineering and conducts research in optical and millimeter wave devices and their applications. Current studies include the millimeter-wave modulation of light and innovation in waveguide gas lasers. He was a Sherman Fairchild Distinguished Scholar at Catech in 1974-1975, and a Visiting Professor at Chalmers Technical University, Göteborg, Sweden in 1989. He is coauthor (with C. K. Birdsall) of Electron Dynamics of Diode Regions (Academic, 1966)

Dr. Bridges is a member of Eta Kappa Nu, Tau Beta Pi, Phi Beta $\mathrm{Kappa}$, and Sigma $\mathrm{Xi}$, receiving Honorable Mention from Eta Kappa Nu as an "Outstanding Young Electrical Engineer" in 1966. He received the Distinguished Teaching Award in 1980 and 1982 from the Associated Students of Caltech. the Arthur L. Schawlow Medal from the Laser Institute of America in 1986, and the IEEE LEOS Quantum Electronics Award in $1988 . \mathrm{He}$ is a member of the National Academy of Engineering and the National Academy of Sciences, and a Fellow of the Optical Society of America and the Laser Institute of America. He has served on various committees of both IEEE and OSA, and was formerly Associate Editor of the IEEE JOURNAL OF QUANTUM ELECTRONICS and the Journal of the Optical Society of America. He was the President of the Optical Society of America in 1988 and a member of the US Air Force Scientific Advisory Board from 1985-1989.

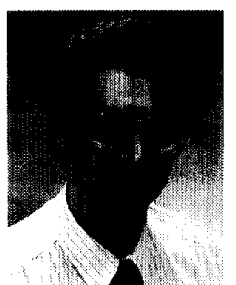

James H. Schaffner (S'74-S'78-M'79-S'80-M'87 $-S^{\prime} 88-M^{\prime} 88$ ) was born in Chicago, IL, on April 27, 1955. He received the B.S., M.S., and Ph.D. degrees from the University of California, Los Angeles, CA, in 1978,1979 , and 1988 , respectively. His doctoral research centered on the high frequency characterization of field effect transistors.

From 1978 to 1988 , he was a Staff Member at Hughes Aircraft Company Missile System Group where he worked on antenna arrays and antenna feed systems for microwave and millimeter wave systems. In addition he was involved with the development of microwave and millimeter wave integrated circuits. Since 1988 he has been with the Hughes Research Laboratories. He is now involved with the development and application of high frequency and high linearity electrooptic and application of high frequency and high linearity electrooptic components for subcarrier multiplexing in analog fiber-optic links. 